\title{
Validity of response assessment criteria in neoadjuvant chemotherapy for gastric cancer (JCOG0507-A)
}

\author{
Yukinori Kurokawa $\cdot$ Taro Shibata \\ Mitsuru Sasako $\cdot$ Takeshi Sano $\cdot$ Akira Tsuburaya • \\ Yoshiaki Iwasaki $\cdot$ Haruhiko Fukuda
}

Received: 14 May 2013/Accepted: 8 August 2013/Published online: 3 September 2013

(C) The International Gastric Cancer Association and The Japanese Gastric Cancer Association 2013

\begin{abstract}
Background Neoadjuvant chemotherapy may improve outcomes in gastric cancer. Tumor responses can be evaluated with RECIST, Japanese Classification of Gastric Carcinoma (JCGC), and histological criteria. These approaches have not yet been compared.

Methods We analyzed two phase II trials of neoadjuvant chemotherapy using S-1 plus cisplatin. JCOG0210 included patients with linitis plastica and large ulcero-invasive tumors, whereas JCOG0405 comprised those with paraaortic or bulky lymph node metastases. Radiologic evaluations were conducted using RECIST in JCOG0405 and JCGC criteria in JCOG0210, because the latter included many patients without measurable lesions. A histological
\end{abstract}

\author{
Y. Kurokawa ( $\square)$ \\ Department of Gastroenterological Surgery, Osaka University \\ Graduate School of Medicine, 2-2-E2 Yamadaoka, \\ Suita Osaka, Japan \\ e-mail: ykurokawa@gesurg.med.osaka-u.ac.jp \\ T. Shibata $\cdot$ H. Fukuda \\ Japan Clinical Oncology Group Data Center, \\ National Cancer Center, Tokyo, Japan \\ M. Sasako \\ Department of Upper Gastrointestinal Surgery, \\ Hyogo College of Medicine, Hyogo, Japan \\ T. Sano \\ Department of Surgery, Cancer Institute Hospital, Tokyo, Japan \\ A. Tsuburaya \\ Department of Surgery, Kanagawa Cancer Center, \\ Kanagawa, Japan \\ Y. Iwasaki \\ Department of Surgery, Tokyo Metropolitan Cancer and \\ Infectious Disease Center Komagome Hospital, Tokyo, Japan
}

responder was defined as a patient in whom one third or more of the tumor was affected. The hazard ratios (HR) for death between responders and non-responders and response rate differences between short- and long-term survivors were estimated.

Results In JCOG0210 $(n=49)$, HR was 0.54 in JCGC responders $(P=0.059)$ and 0.40 in histological responders $(P=0.005)$. The difference in response rates between short- and long-term survivors using histological criteria (34 \%, $P=0.023$ ) was greater than that using JCGC criteria $(24 \%, P=0.15)$. In JCOG0405 $(n=51)$, HR was 0.67 in RECIST responders $(P=0.35)$ and 0.39 in histological responders $(P=0.030)$. In short- and long-term survivors, respectively, RECIST response rates were 62 and $67 \%(P=0.77)$, whereas histological response rates were 33 and $63 \%(P=0.048)$.

Conclusions Histological criteria showed higher response assessment validity than RECIST or JCGC criteria and yielded the best surrogate endpoint for overall survival.

Keywords RECIST $\cdot$ JCGC $\cdot$ JCOG

\section{Introduction}

Gastric cancer is the second-leading cause of cancer deaths worldwide and the most common cancer in Japan and Korea [1]. Although surgery is the standard treatment for resectable gastric cancer [2,3], the prognosis of patients with advanced tumors is poor [4]. In particular, linitis plastica (Borrmann type 4) and large ulcero-invasive-type (Borrmann type 3) tumors, as well as those with paraaortic nodal metastases or bulky lymph node metastases, have extremely poor outcomes even after curative resection [5, 6]. For these advanced tumors, neoadjuvant chemotherapy 
is expected to improve long-term prognoses. The Japan Clinical Oncology Group (JCOG) has conducted two multicenter phase II trials to evaluate the efficacy and safety of S-1 plus cisplatin as a neoadjuvant regimen and has reported promising results $[7,8]$.

Three criteria for evaluating tumor responses to chemotherapy are currently available. The Response Evaluation Criteria in Solid Tumors (RECIST) is the gold standard in the evaluation of tumor response, but it requires the presence of a measurable lesion [9]. Because resectable gastric cancer seldom has measurable lesions, we cannot use RECIST in the neoadjuvant setting. The Japanese Classification of Gastric Carcinoma (JCGC) includes a response evaluation criterion involving barium X-ray or endoscopic examination, which is useful for tumors without measurable lesions [10]. Furthermore, we can evaluate tumor response histologically in resected specimens. As there have been no studies comparing the validities of these radiologic and histological criteria, we have conducted this correlative study (JCOG0507-A) to find the best surrogate endpoint for overall survival in neoadjuvant studies for gastric and esophageal cancers. In esophageal cancer patients, we had reported that histological response rate was the better surrogate endpoint for survival than RECIST response rate [11]. This article reports the results for gastric cancer, comparing RECIST, JCGC, and histological criteria.

\section{Methods}

Patient population

We included all eligible patients from two clinical trials (JCOG0210 and JCOG0405) that were conducted by the JCOG. These phase II trials aimed to evaluate the efficacy and safety of neoadjuvant S-1 plus cisplatin in gastric cancer patients [7, 8]. The eligibility criteria of the JCOG0210 trial included linitis plastica (Borrmann type 4) and ulcero-invasive-type (Borrmann type 3) tumors. In the case of ulcero-invasive tumors, the size of the primary tumor was required to be $8 \mathrm{~cm}$ or larger. Between March 2003 and December 2003, 49 eligible patients were enrolled in the JCOG0210 trial. Responses to neoadjuvant chemotherapy were evaluated using JCGC criteria. After preoperative chemotherapy, 41 patients could undergo gastrectomy, which provided tissue samples for use in assessing the histological response to preoperative chemotherapy. Six patients failed in simple laparotomy because of the presence of incurable lesions. Two patients did not undergo surgery for reasons of chemotherapyrelated death in one patient and refusal of any protocol treatment in one patient.
The eligibility criteria for the JCOG0405 trial included gastric cancer with paraaortic nodal metastases or bulky lymph nodes. Between February 2005 and June 2007, 51 eligible patients were enrolled in the JCOG0405 trial. Responses to neoadjuvant chemotherapy were evaluated with RECIST. After preoperative chemotherapy, 48 patients could undergo gastrectomy, which provided material to evaluate the histological response to preoperative chemotherapy. Three patients did not undergo surgery because of progressive tumor.

All patients in the JCOG0210 and the JCOG0405 trials gave written informed consent. These trials were approved by the JCOG Clinical Trial Review Committee and the institutional review board of each institution involved. Permission for the secondary use of trial data was included in patients' informed consent for JCOG0210 and JCOG0405. The protocol of this correlative study (JCOG0507-A) was approved by the JCOG Protocol Review Committee. JCOG0405 is registered with UMINCTR (http://www.umin.ac.jp/ctr/), identification number C000000094.

\section{Treatments}

The same chemotherapy regimen was used in each of the two trials. S-1 was given orally at $80 \mathrm{mg} / \mathrm{m}^{2}$ for the first 3 weeks of a 4-week cycle. Cisplatin was given as an intravenous infusion of $60 \mathrm{mg} / \mathrm{m}^{2}$ on day 8 of each cycle. Patients received two 4-week cycles of neoadjuvant S-1 plus cisplatin and then underwent gastrectomy with D2 (in the JCOG0210 trial) or D2 plus paraaortic lymphadenectomy (in the JCOG0405 trial). If curative resection was considered difficult after the second course, addition of a further course of chemotherapy before surgery was permitted only in the JCOG0405 trial. After surgery, no further treatment was given until tumor recurrence.

\section{Response evaluation}

After the second course of neoadjuvant chemotherapy, tumor response was evaluated with JCGC criteria based on computed tomography (CT), barium X-ray, and endoscopic examination findings in the JCOG0210 trial, whereas response evaluation using RECIST in the JCOG0405 trial was based only on CT findings. These evaluations were performed by the central reviewers. Response evaluations based on RECIST were not conducted in the JCOG0210 trial because many patients did not have measurable lesions. We could not evaluate the JCGC response in the JCOG0405 trial because barium X-rays and endoscopic examinations were not performed after neoadjuvant chemotherapy. 
With the JCGC criteria, overall tumor response depends on the combined responses of primary gastric lesions and metastatic lesions. The details of the JCGC criteria have been described elsewhere [10]. Briefly, morphological changes of gastric lesions are evaluated by X-ray or endoscopic examinations, and the overall responses are classified into four categories: complete response (CR), partial response (PR), no change (NC), or progressive disease (PD). Measurable lesions with at least a $50 \%$ decrease in total tumor size in two dimensions and at least a $30 \%$ decrease in total tumor size in one dimension are classified as PR. Evaluable but nonmeasurable lesions with flattening on X-ray or endoscopic examination, or diffusely infiltrating lesions with at least $50 \%$ enlargement of the gastric lumen in the tumor area by X-ray examination, are also classified as PR. CR or PR cases were treated as responders.

Surgical specimens were assessed histologically, and tumor response was evaluated according to the histological criteria of the JCGC [12]. Briefly, histological evaluations were classified into five categories according to the proportion of the tumor affected by degeneration or necrosis: grade 3 , no viable tumor cells remain; grade 2 , viable tumor cells remain in less than $1 / 3$ of the tumorous area; grade $1 \mathrm{~b}$, viable tumor cells remain in more than $1 / 3$ but less than $2 / 3$ of the tumorous area; grade $1 \mathrm{a}$, viable tumor cells occupy more than $2 / 3$ of the tumorous area; grade 0 , no evidence of treatment effect. A histological responder was defined as a patient in whom one third or more of the tumor was affected (grade 1b, 2, or 3). Because the definition of histological responder is controversial, we also evaluated the results when a histological responder was classified as grade 2 or 3 . Patients who did not undergo surgery were regarded as non-responders. These evaluations were performed by the pathologists at each institution.

\section{Statistical analysis}

The data from all eligible patients were analyzed in this study. Cases in which the tumor was not resected or could not be evaluated were treated as non-responders. With the methods used in this study, a comparison of the overall survival between responders and non-responders was said to have a pitfall because early death cases were classified into the non-responder group. In our study, however, there were no early deaths during the protocol treatment, which implies that minimal bias was induced by the classification system employed in our study.

The relationship of response and overall survival was evaluated using hazard ratios (HRs). The HR for death of responders to non-responders was estimated using the Cox proportional hazard model, and survival distributions were compared using the log-rank test. The difference in response rates between short- and long-term survivors was estimated and tested with Fisher's exact test. Statistical analysis was performed with SAS version 9.2 (SAS Institute, Cary, NC, USA).

\section{Results}

Patient characteristics

The clinicopathological characteristics of all eligible patients in the JCOG 0210 and 0405 trials are shown in Table 1. The proportion of male patients in the JCOG0405 trial was higher than that in the JCOG0210 trial. The majority of tumors were of the undifferentiated type in the JCOG0210 trial, whereas the differentiated type was more frequent in the JCOG0405 trial. Pathological node-negative (pN0) patients comprised $16 \%$ of both the JCOG0210 (8 of 49) and JCOG0405 (8 of 51) trial populations.

\section{Response rates}

The responses to neoadjuvant $\mathrm{S}-1$ plus cisplatin as evaluated by the JCGC, RECIST, and histological criteria are shown in Table 1. The response rates in the JCOG0210 trial were $57 \%$ [95\% confidence interval (CI), 42-71\%] with the JCGC criteria and $47 \%$ (95\% CI, 33-62\%) with histological criteria. The response rates in the JCOG0405 trial were $65 \%$ (95\% CI, 50-78\%) with the RECIST and $51 \%(95 \%$ CI, 37-65\%) with histological criteria.

\section{Survival curves in responders and non-responders}

Overall survival curves for the JCOG0210 trial are shown in Fig. 1. The difference of the 3-year overall survival rate between responders and non-responders was $17.8 \%$ (responders, $32.1 \%$; non-responders, $14.3 \%$ ) on JCGC criteria and $27.6 \%$ (responders, 39.1\%; non-responders, $11.5 \%$ ) on histological criteria. The HR for death of histological responders to non-responders $(0.40 ; 95 \% \mathrm{CI}$, $0.20-0.77)$ was lower than that using JCGC criteria $(0.54$; $95 \%$ CI, 0.28-1.03), and the log-rank $P$ value on histological criteria $(P=0.005)$ was much smaller than that on JCGC criteria $(P=0.059)$.

Overall survival curves for the JCOG0405 trial are shown in Fig. 2. The difference in the 3-year overall survival rate between responders and non-responders was $5.0 \%$ (responders, $60.6 \%$; non-responders, $55.6 \%$ ) on RECIST and $29.1 \%$ (responders, $73.1 \%$; non-responders, $44.0 \%$ ) on histological criteria. The HR for death of histological responders to non-responders $(0.39 ; 95 \% \mathrm{CI}$, 0.17-0.94) was lower than that using the RECIST $(0.67$; 
Table 1 Patient characteristics

\begin{tabular}{|c|c|c|}
\hline & $\begin{array}{l}\text { JCOG0210 } \\
(n=49)\end{array}$ & $\begin{array}{l}\text { JCOG0405 } \\
(n=51)\end{array}$ \\
\hline \multicolumn{3}{|l|}{ Age (years) } \\
\hline Median & 61 & 63 \\
\hline Range & $32-75$ & $42-75$ \\
\hline \multicolumn{3}{|l|}{ Gender } \\
\hline Male & 28 & 42 \\
\hline Female & 21 & 9 \\
\hline \multicolumn{3}{|c|}{ Borrmann macroscopic type } \\
\hline 0 & 0 & 2 \\
\hline 1 & 0 & 1 \\
\hline 2 & 0 & 15 \\
\hline 3 & 20 & 31 \\
\hline 4 & 29 & 0 \\
\hline 5 & 0 & 2 \\
\hline \multicolumn{3}{|l|}{ Histology } \\
\hline Differentiated & 9 & 28 \\
\hline Undifferentiated & 40 & 22 \\
\hline Unknown & 0 & 1 \\
\hline \multicolumn{3}{|l|}{ Clinical $\mathrm{T}$ stage } \\
\hline $\mathrm{cT} 1$ & 0 & 1 \\
\hline $\mathrm{cT} 2$ & 2 & 10 \\
\hline cT3 & 45 & 38 \\
\hline cT4 & 2 & 2 \\
\hline \multicolumn{3}{|l|}{ Clinical N stage } \\
\hline $\mathrm{cNO}$ & 16 & 0 \\
\hline $\mathrm{cN} 1$ & 20 & 0 \\
\hline $\mathrm{cN} 2$ & 13 & 25 \\
\hline $\mathrm{cN} 3$ & 0 & 26 \\
\hline \multicolumn{3}{|l|}{ Residual tumor } \\
\hline R0 & 31 & 42 \\
\hline $\mathrm{R} 1$ or $\mathrm{R} 2$ & 10 & 6 \\
\hline Unresected & 8 & 3 \\
\hline \multicolumn{3}{|l|}{ Pathological $\mathrm{T}$ stage } \\
\hline pT0 & 1 & 2 \\
\hline pT1 & 4 & 7 \\
\hline pT2 & 11 & 23 \\
\hline pT3 & 18 & 15 \\
\hline pT4 & 7 & 1 \\
\hline Unresected & 8 & 3 \\
\hline \multicolumn{3}{|l|}{ Pathological N stage } \\
\hline pNO & 8 & 8 \\
\hline $\mathrm{pN} 1$ & 10 & 5 \\
\hline $\mathrm{pN} 2$ & 16 & 21 \\
\hline $\mathrm{pN} 3$ & 7 & 14 \\
\hline Unresected & 8 & 3 \\
\hline \multicolumn{3}{|c|}{ Tumor responses evaluated by the JCGC criteria } \\
\hline $\mathrm{CR}$ & 0 & - \\
\hline PR & 28 & - \\
\hline
\end{tabular}

Table 1 continued

\begin{tabular}{lll}
\hline & $\begin{array}{l}\text { JCOG0210 } \\
(n=49)\end{array}$ & $\begin{array}{l}\text { JCOG0405 } \\
(n=51)\end{array}$ \\
\hline NC & 13 & - \\
PD & 3 & - \\
NE & 5 & - \\
Tumor responses evaluated by the RECIST & \\
CR & - & 0 \\
PR & - & 33 \\
SD & - & 14 \\
PD & - & 4
\end{tabular}

Tumor responses evaluated by the histological criteria of the JCGC

$\begin{array}{lll}\text { Grade } 3 & 1 & 1 \\ \text { Grade } 2 & 12 & 13 \\ \text { Grade } 1 \mathrm{~b} & 10 & 12 \\ \text { Grade } 1 \mathrm{a} & 9 & 19 \\ \text { Grade } 0 & 9 & 3 \\ \text { Unresected } & 8 & 3\end{array}$

$\mathrm{T}$ stage and $\mathrm{N}$ stage were according to the 13th edition of the Japanese Classification of Gastric Carcinoma

$J C G C$ Japanese classification of gastric carcinoma, $C R$ complete response, $P R$ partial response, $N C$ no change, $S D$ stable disease, $P D$ progressive disease, $N E$ not evaluable

$95 \% \mathrm{CI}, 0.29-1.56)$, and the log-rank $P$ value on histological criteria $(P=0.030)$ was much smaller than that on RECIST $(P=0.35)$.

When a histological responder was classified as grade 2 or 3 , the HRs for death of modified histological responders to non-responders were 0.57 (95\% CI, 0.26-1.25) in the JCOG0210 trial and 0.32 (95\% CI, 0.09-1.08) in the JCOG0405 trial. The log-rank $P$ values on modified histological criteria were 0.15 in the JCOG0210 and 0.067 in the JCOG0405.

Response rates in short- and long-term survivors

Because the median overall survival time in all eligible patients in the JCOG0210 was 17.3 months, we divided patients into short- and long-term survivors with a cutoff for overall survival time of 18 months. The respective response rates based on JCGC and histological criteria were $46 \%$ and $31 \%$ in short-term survivors and $70 \%$ and $65 \%$ in long-term survivors (Fig. 3). The difference in response rates between short- and long-term survivors using histological criteria (Fisher's exact test, $P=0.023$ ) was greater than that using JCGC criteria (Fisher's exact test, $P=0.15$ ).

Although for the JCOG0405 trial the median overall survival time was not reached at the time of this analysis, the 3 -year overall survival rate was $59 \%$. We therefore set 
Fig. 1 Overall survival curves between responders and nonresponders for the JCOG0210 trial: JCGC criteria (a), $P=0.059$ (log-rank test); histological criteria (b), $P=0.005$ (log-rank test)

\section{a JCGC criteria}

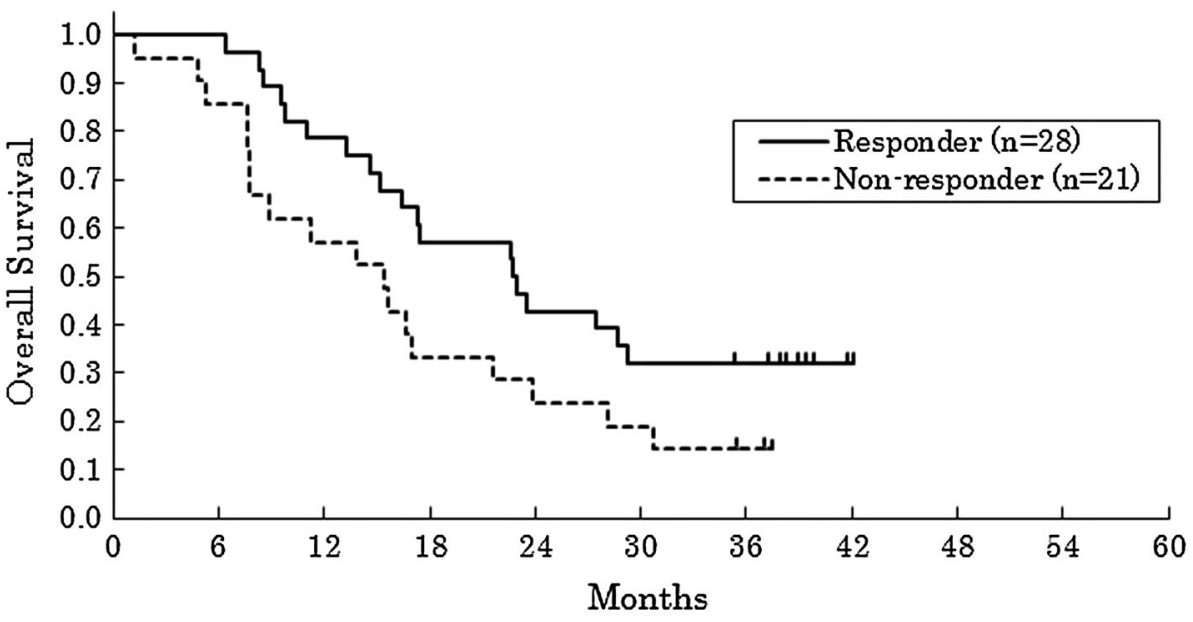

b histological criteria

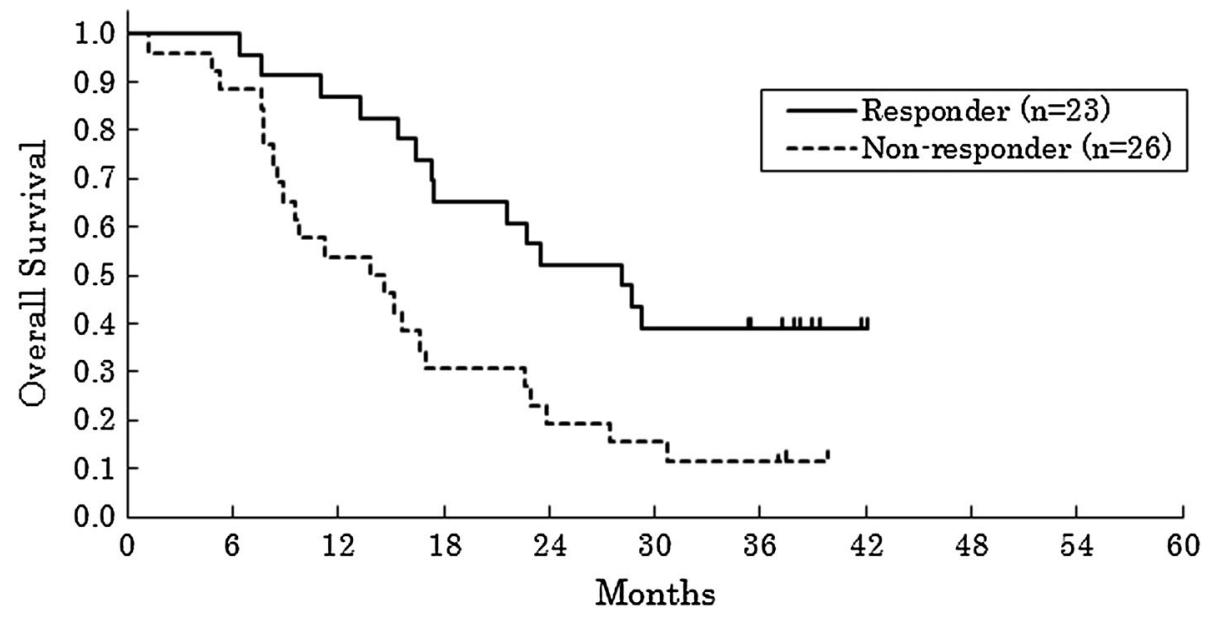

the cutoff for overall survival time at 36 months. The respective response rates using RECIST and histological response rates were $62 \%$ and $33 \%$ in short-term survivors and $67 \%$ and $63 \%$ in long-term survivors (Fig. 4). The difference in response rates between short- and long-term survivors using histological criteria (Fisher's exact test, $P=0.048$ ) was greater than that using RECIST (Fisher's exact test, $P=0.77$ ).

Again, when a histological responder was classified as grade 2 or 3 , the differences in response rates between short- and long-term survivors based on modified histological criteria were $16 \%$ (Fisher's exact test, $P=0.33$ ) in the JCOG0210 trial and $22 \%$ (Fisher's exact test, $P=0.11)$ in the JCOG0405 trial.

\section{Discussion}

In this correlative study of two phase II trials, histological criteria, as compared to RECIST and JCGC criteria, demonstrated a greater difference in both overall survival between responders and non-responders and in response rates between short- and long-term survivors. This result indicates that the histological response was the best surrogate endpoint for overall survival in these neoadjuvant trials for gastric cancer, and the conclusion for gastric cancer was the same as that in esophageal cancer [11], which is very important for the development of cancer treatments. If the histological response can be used as the primary endpoint in neoadjuvant settings, we can evaluate any gastric cancer population regardless of the presence of measurable lesions.

RECIST is the gold standard in the evaluation of tumor responses, but it requires the presence of a measurable lesion. In the present version of RECIST (Ver. 1.1), the criteria for measurable lesions were revised to be stricter: a lymph node must be more than $15 \mathrm{~mm}$ in short-axis diameter [13]. There are many unresectable gastric cancer patients without measurable metastatic lesions, because the most frequent pattern of recurrence in advanced or 
Fig. 2 Overall survival curves between responders and nonresponders for the JCOG0405 trial: RECIST (a), $P=0.35$ (log-rank test); histological criteria (b), $P=0.030(\log$ rank test)
Fig. 3 Comparison of the response rates between shortterm (short survivors) and longterm (long survivors) survivors for the JCOG0210 trial: JCGC criteria (a), $P=0.15$ (Fisher's exact test); histological criteria (b), $P=0.023$ (Fisher's exact test)

\section{a RECIST}

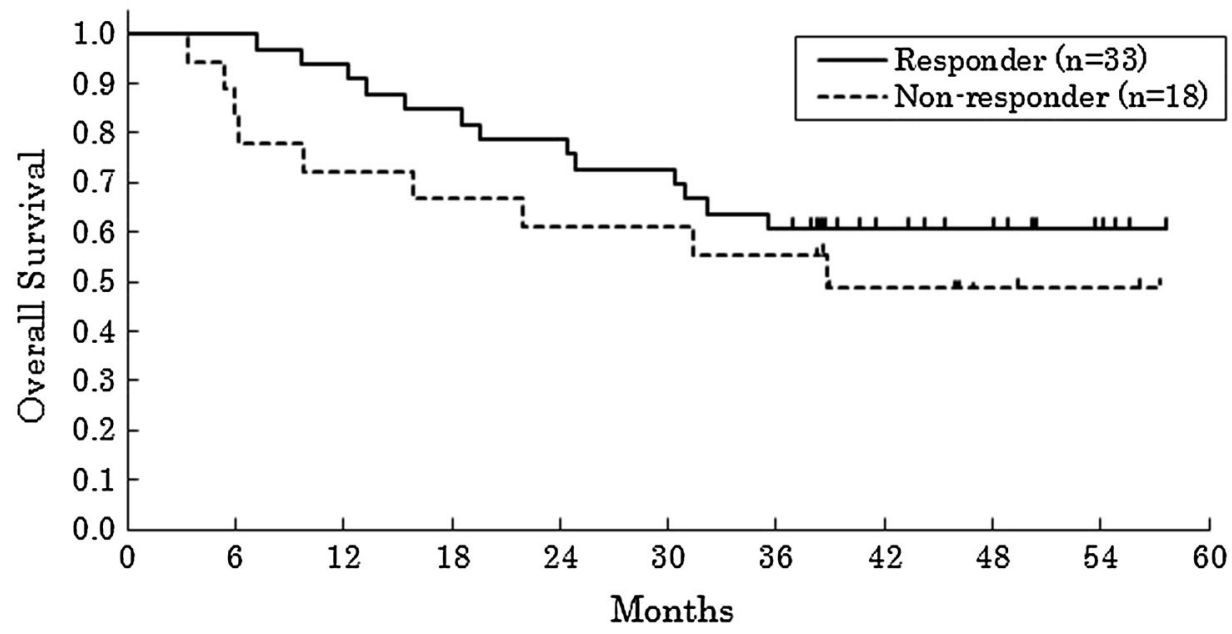

b histological criteria

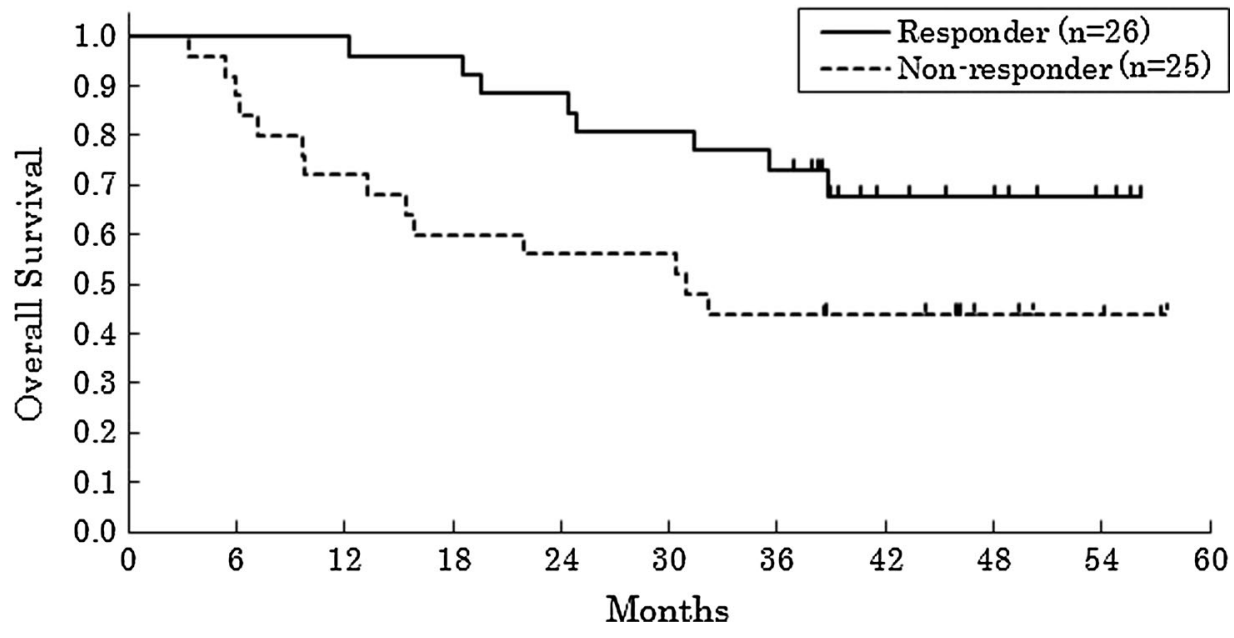

a JCGC criteria

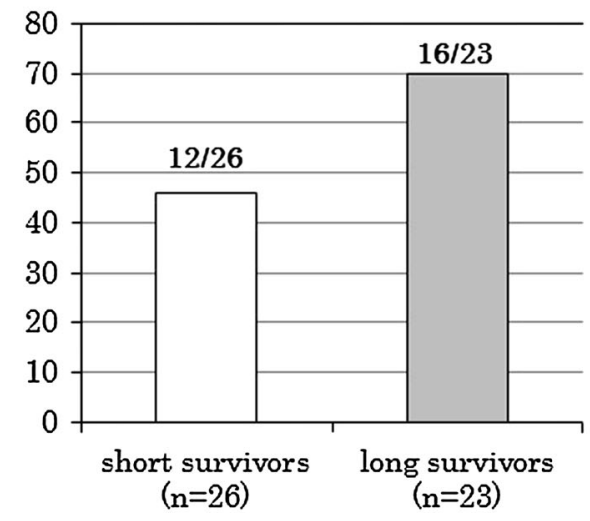

\section{b histological criteria}

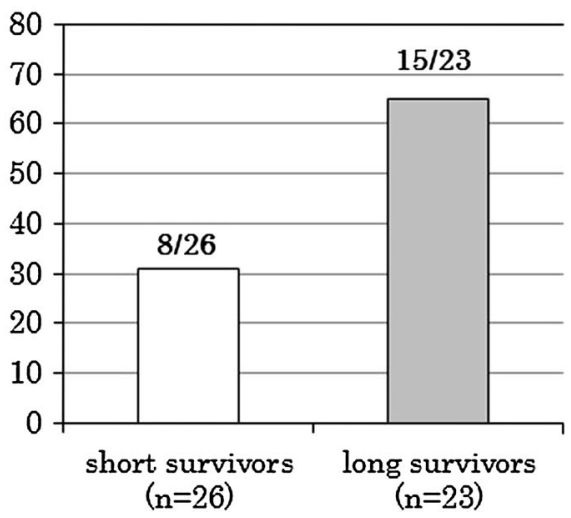

recurrent cases is peritoneal seeding. Particularly in neoadjuvant settings, resectable gastric tumors seldom have measurable lesions. Furthermore, the primary lesion of digestive tract is not suitable for measurable lesion in terms of reproducibility, as the RECIST guideline cautioned.
The JCGC response evaluation criteria were established to evaluate tumor responses even for tumors without measurable lesions. Although it can be used for any type of gastric cancer, evaluation using endoscopic examination is subjective. Furthermore, repetition of barium X-ray or 
Fig. 4 Comparison of the response rates between shortand long-term survivors for the JCOG0405 trial: RECIST (a), $P=0.77$ (Fisher's exact test); histological criteria (b), $P=0.048$ (Fisher's exact test)

\section{a RECIST}

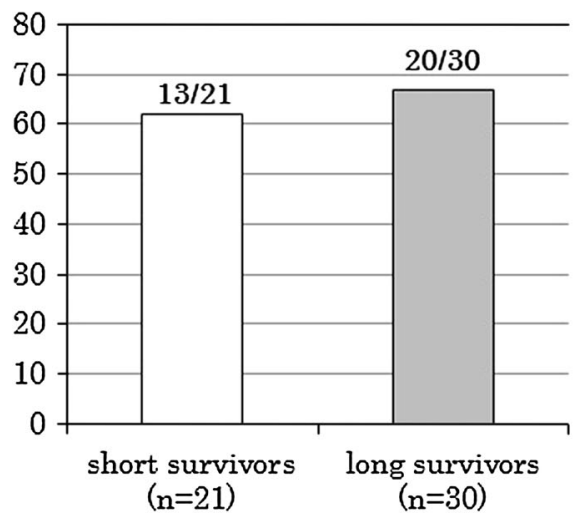

\section{b histological criteria}

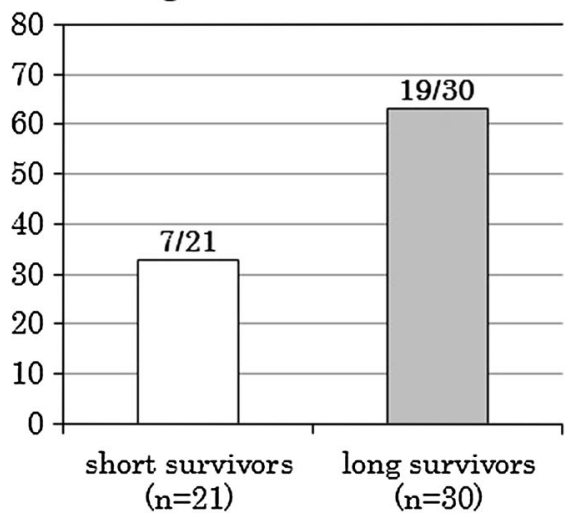

endoscopic examination for evaluation of tumor response is a significant burden for patients. In contrast, histological evaluation does not require any presurgical examination. If the histological response is indeed the most useful indicator in neoadjuvant settings, patients will not need to undergo invasive examinations after chemotherapy.

There are several different histological grading systems for the evaluation of tumor responses in addition to that defined by the JCGC. Becker et al. [14, 15] proposed the following system: tumors with no viable cells are assigned grade Ia; tumors with $1-10 \%$ viable cells, grade Ib; tumors with $10-50 \%$ viable cells, grade II; and tumors with more than $50 \%$ viable cells, grade III. Ajani et al. [16, 17] proposed a grading scheme: cases showing either an absence of tumor cells or necrosis in more than $90 \%$ of the resected tumor were classified as responders. In this study, we used the JCGC grading system, whereby cases showing viable tumor cells remain in less than two thirds are classified as responders, but for our sensitivity analysis we changed the cutoff point from two thirds to one third. Both the JCOG0210 and JCOG0405 trials showed similar results using this grading system.

This study had some limitations. First, histological evaluations were performed only by the pathologists at each institution, although response evaluations using RECIST and JCGC criteria were conducted by the central reviewers. Because histological evaluations are not completely objective, there may have been some issues with inter-rater reliability. However, our JCOG study group institutions are staffed with experts not only in surgery and chemotherapy, but also in pathology. We believe there was little heterogeneity in the histological evaluations performed by this experienced group. Another study is now ongoing to compare the predictive values based on the different scoring systems of histological response after central review by two reference pathologists. A second limitation is that this study enrolled only patients who had received preoperative S-1 plus cisplatin. S-1 plus cisplatin is one of the standard regimens for metastatic gastric cancer $[18,19]$. The validity of histological tumor response evaluation may vary with different chemotherapeutic regimens, and further studies are needed to investigate this point.

In conclusion, histological response rate seemed to be a better surrogate endpoint for overall survival than radiologic response rate in studies of neoadjuvant therapy for gastric cancer.

Acknowledgments We thank Harumi Kaba for data management, Junki Mizusawa for statistical support, and Dr. Hiroshi Katayama and Dr. Kenichi Nakamura for reviewing the manuscript. This study was supported by National Cancer Center Research and Development Fund (23-A-16 and 23-A-19), Grants-in-Aid for Cancer Research (14S-3, 14S-4, 17S-3, 17S-5, 20S-3, and 20S-6), from the Ministry of Health, Labour and Welfare of Japan.

\section{References}

1. GLOBOCAN. http://globocan.iarc.fr/ (2008).

2. Sasako M, Sano T, Yamamoto S, Sairenji M, Arai K, Kinoshita $\mathrm{T}$, et al. Left thoracoabdominal approach versus abdominaltranshiatal approach for gastric cancer of the cardia or subcardia: a randomised controlled trial. Lancet Oncol. 2006;7:644-51.

3. Sasako M, Sano T, Yamamoto S, Kurokawa Y, Nashimoto A, Kurita A, et al. D2 lymphadenectomy alone or with para-aortic nodal dissection for gastric cancer. N Engl J Med. 2008;359:453-62.

4. Nashimoto A, Akazawa K, Isobe Y, Miyashiro I, Katai H, Kodera Y, et al. Gastric cancer treated in 2002 in Japan: 2009 annual report of the JGCA nationwide registry. Gastric Cancer. 2013;16:1-27.

5. Kinoshita T, Sasako M, Sano T, Katai H, Furukawa H, Tsuburaya A, et al. Phase II trial of S-1 for neoadjuvant chemotherapy against scirrhous gastric cancer (JCOG 0002). Gastric Cancer. 2009;12:37-42.

6. Keighley MR, Moore J, Roginski C, Powell J, Thompson H. Incidence and prognosis of $\mathrm{N} 4$ node involvement in gastric cancer. Br J Surg. 1984;71:863-6.

7. Iwasaki Y, Sasako M, Yamamoto S, Nakamura K, Sano T, Katai $\mathrm{H}$, et al. Phase II study of preoperative chemotherapy with S-1 and cisplatin followed by gastrectomy for clinically resectable type 4 and large type 3 gastric cancers (JCOG0210). J Surg Oncol 2013;107(7):741-745 
8. Yoshikawa T, Nakamura K, Tsuburaya A, Sano T, Mizusawa J, Katai $\mathrm{H}$, et al. A phase II study of preoperative chemotherapy with S-1 (S) and cisplatin (P) followed by D3 gastrectomy for gastric cancer (GC) with extensive lymph node metastasis (ELM): survival results of JCOG0405. J Clin Oncol 2011;29(suppl 4): abstr 70

9. Therasse P, Arbuck SG, Eisenhauer EA, Wanders J, Kaplan RS, Rubinstein L, et al. New guidelines to evaluate the response to treatment in solid tumors. European Organization for Research and Treatment of Cancer, National Cancer Institute of the United States, National Cancer Institute of Canada. J Natl Cancer Inst. 2000;92:205-16.

10. Japanese Gastric Cancer Association. Japanese classification of gastric carcinoma, 2nd English edition. Response assessment of chemotherapy and radiotherapy for gastric carcinoma: clinical criteria. Gastric Cancer. 2001;4:1-8.

11. Kurokawa Y, Shibata T, Ando N, Seki S, Mukaida H, Fukuda H. Which is the optimal response criteria for evaluating preoperative treatment in esophageal cancer: RECIST or histology? Ann Surg Oncol. 2013;20:3009-14.

12. Japanese Gastric Cancer Association. Japanese classification of gastric carcinoma, 3rd English edition. Gastric Cancer. 2011;14:101-12.

13. Eisenhauer EA, Therasse P, Bogaerts J, Schwartz LH, Sargent D, Ford R, et al. New response evaluation criteria in solid tumours: revised RECIST guideline (version 1.1). Eur J Cancer. 2009;45: $228-47$.
14. Becker K, Fumagalli U, Mueller JD, Fink U, Siewert JR, Hofler H. Neoadjuvant chemotherapy for patients with locally advanced gastric carcinoma: effect on tumor cell microinvolvement of regional lymph nodes. Cancer (Phila). 1999;85:1484-9.

15. Becker K, Mueller JD, Schulmacher C, Ott K, Fink U, Busch R, et al. Histomorphology and grading of regression in gastric carcinoma treated with neoadjuvant chemotherapy. Cancer (Phila). 2003;98:1521-30.

16. Ajani JA, Mansfield PF, Janjan N, Morris J, Pisters PW, Lynch $\mathrm{PM}$, et al. Multi-institutional trial of preoperative chemoradiotherapy in patients with potentially resectable gastric carcinoma. J Clin Oncol. 2004;22:2774-80.

17. Ajani JA, Mansfield PF, Crane CH, Wu TT, Lunagomez S, Lynch $\mathrm{PM}$, et al. Paclitaxel-based chemoradiotherapy in localized gastric carcinoma: degree of pathologic response and not clinical parameters dictated patient outcome. J Clin Oncol. 2005;23:1237-44.

18. Koizumi W, Narahara H, Hara T, Takagane A, Akiya T, Takagi M, et al. S-1 plus cisplatin versus S-1 alone for first-line treatment of advanced gastric cancer (SPIRITS trial): a phase III trial. Lancet Oncol. 2008;9:215-21.

19. Ajani JA, Rodriguez W, Bodoky G, Moiseyenko V, Lichinitser $\mathrm{M}$, Gorbunova V, et al. Multicenter phase III comparison of cisplatin/S-1 with cisplatin/infusional fluorouracil in advanced gastric or gastroesophageal adenocarcinoma study: the FLAGS trial. J Clin Oncol. 2010;28:1547-53. 